\title{
Cerebral lateralization and general intelligence: Gender differences in a transcranial Doppler study
}

\author{
Philip C. Njemanze ${ }^{*}$ \\ Non-invasive Neurocybernetic Flow Laboratory, International Institutes of Advanced Research and Training, \\ Chidicon Medical Center, Owerri, Imo State, Nigeria \\ Accepted 14 June 2004 \\ Available online 4 August 2004
}

\begin{abstract}
The present study evaluated cerebral lateralization during Raven's progressive matrices (RPM) paradigm in female and male subjects. Bilateral simultaneous transcranial Doppler (TCD) ultrasound was used to measure mean blood flow velocities (MBFV) in the right and left middle cerebral arteries (MCAs) in 24 (15 females and 9 males) right-handed normal subjects. The female subjects used a left hemisphere strategy, while males used a right hemisphere strategy to successfully solve RPM tasks. This implies that general intelligence is associated with neural systems within one hemisphere that are accessible to a variety of cognitive processes.

(c) 2004 Elsevier Inc. All rights reserved.
\end{abstract}

Keywords: Cognition; Language; Cerebral blood flow; Ultrasound; Brain; Sex; Psychological tests; Laterality

\section{Introduction}

The determinants of human performance have been a subject of intense debate for over a century. Spearman in 1904, put forward the idea that measures of performance or success in diverse cognitive tests show a pattern of almost universal positive correlation (Jensen, 1987; Spearman, 1904, 1923, 1927). He postulated the hypothesis of a general intelligence or $g$ factor making some contribution to success in diverse forms of cognitive activity. Subsequently, two contrasting postulates on cortical localization of intelligence were put forward. The first proposes that, processing of any task receives contribution from a large set of components or information-processing functions (Lashley, 1921, 1929; Satz, Strauss, \& Whitaker, 1990; Thomson, 1951; Thurstone, 1938, 1940). Therefore, any two tasks are likely to share at least some components, giving rise to universal posi-

\footnotetext{
${ }^{*}$ Fax: +234-83-23-11-83.

E-mail address: chidicon@yahoo.com.
}

tive correlation. This hypothesis assumes that, general ability is diffusely represented on the whole cortex, and injury to any region of the brain produces an intellectual decrement. Conversely, the second, so-called hierarchic hypothesis postulates that, in the course of evolution some area of the brain has gained a dominant role in sustaining general intelligence (Basso, De Renzi, Faglioni, Scotti, \& Spinnler, 1973).

Snow, Kyllonen, and Marshalek (1984) have classified intelligence tests by task complexity, into tests at the center and tests at the periphery. Psychometric tests including RPM (Raven, 1938) and other complex reasoning tests were classified as tests at the center, while simpler tests were placed at the periphery. Applying this construct, irrespective of the views held by both hypotheses, the centrality of the RPM emerges in either case.

Furthermore, it has been suggested that RPM is a good test of intelligence, and should account for a great deal of the reasoning in other tests (Carpenter, Just, \& Shell, 1990). It has been suggested that the neural substrate for intelligence lies within prefrontal cortex (Prabhakaran, Smith, Desmond, Glover, \& Gabrieli, 1997), and 
post-rolandic structures (Basso et al., 1973). Recently, positron emission tomography studies have shown selective recruitment of lateral prefrontal cortex in one or both hemispheres (Duncan et al., 2000). Studies of cerebral lateralization of intelligence may implicate one hemisphere, or both, if general abilities were diffusely represented.

Cerebral lateralization could be studied using TCD (Bulla-Hellweg, Vollmer, Götzen, Skreczek, \& Hartje, 1996; Cupini et al., 1996; Droste, Harders, \& Rastogi, 1989; Evers, Dannert, Rodding, Rotter, \& Ringelstein, 1999; Hartje, Ringelstein, Kistinger, Fabianek, \& Willmes, 1994; Kelley et al., 1992; Markus \& Boland, 1992; Njemanze, 1991, 1996, 2002, 2004; Njemanze, Gomez, \& Horenstein, 1992; Rihs, Gutbrod, Steiger, Sturzenegger, \& Mattle, 1995; Silvestrini, Troisi, Matteis, Razzano, \& Caltagirone, 1994; Varnadore, Roberts, \& McKinney, 1997; Vingerhoets \& Stroobant, 1999). Studies with TCD have been cross-validated by functional MRI (Schmidt et al., 1999), and reproducibility assessed (Knecht et al., 1998).

The purpose of the present study is to evaluate cerebral lateralization during RPM tasks in females and males. It is postulated that general intelligence is associated with neural systems represented in one hemisphere that are accessible to a variety of cognitive processes. Consequently, unilateral representation of general intelligence will cause MBFV changes in ipsilateral MCA during performance of intelligence tasks. More specifically, the present study evaluates MBFV changes in both MCAs during performance of RPM tasks in both female and male subjects.

\section{Materials and methods}

\subsection{Subjects}

The studied group included 24 normal volunteers, 15 female and 9 male. Mean age was $25.08 \pm 3.16$ years (range 21-33); females (25.47 \pm 3.5 years) did not differ from males $(24.44 \pm 2.5$ years). All were right-handed as determined using the Edinburgh handedness inventory (mean laterality quotient was 100) (Oldfield, 1971). Subjects had no history of neurological or cardiovascular or respiratory diseases. None was under medication, including contraceptive pills for females or recreational drugs. All refrained from ingesting caffeine at least $24 \mathrm{~h}$ prior to the study. The years of schooling ranged from 16 to 18 . All subjects signed informed consent for the study. The Institutional Ethical Committee approved the study protocol.

\subsection{Normal resting baseline measurements}

Baseline data acquisition were made with subject in supine horizontal position with head inclined up at $30^{\circ}$. The eyes were fixated on a cube target, projected onto a screen, placed $80 \mathrm{~cm}$ (from the nasal ridge) in front of the subject. The ears were partially closed with earplugs that also provided the base of the probe holder. All environmental noise including sound from the TCD instrument was excluded, and environmental luminance was kept constant for all participants. Measurements comprising a $60 \mathrm{~s}$ continuous train of velocity waveform envelopes were recorded with subject mute, still, and attention focused on the cube with no mental or manual task to perform.

\subsection{Raven's progressive matrices test}

Eight successive black and white slides of RPM tasks were arranged in increasing levels of difficulty and sequentially projected on a screen. Subjects were required to determine which of the 6-8 options best completes the matrix, so that the inter-relational rules among the elements between the row and columns were met. No verbal answers were required, instead subjects were asked to indicate their choice of option by slightly raising the corresponding finger during the inter-stimulus interval. The fingers were numbered sequentially from 1 to 8 , starting with the right thumb as the first option to the left middle finger as option eight. An observer recorded the option signed by the subject during the 2 s inter-stimulus interval, also used for task slide transition. All responses were transformed into Wrong or Correct ANSWER. Measurements comprising a $60 \mathrm{~s}$ continuous train of velocity waveform envelopes were recorded during each task with subject mute, still and attention focused on the task.

\subsection{Transcranial Doppler measurement}

Transcranial Doppler scanning was performed by a method similar to that previously described elsewhere (Njemanze, 1991, 1996, 2002), using a bilateral simultaneous TCD instrument (Multi-Dop T, DWL, Sipplingen, Germany). MBFV in both MCAs was sampled in supine horizontal position using two $2 \mathrm{MHz}$ transducers, with probes fixated on both temples using a probe holder-LAM-Rack (DWL, Sipplingen, Germany). The Doppler signals and a continuous train of flow velocity envelops were obtained at a depth of $50 \mathrm{~mm}$ from the probe surface with the same gain and power settings for each subject. MBFV was recorded and averaged in $10 \mathrm{~s}$ segments for each condition. Subjects were instructed to remain mute and motionless, and the probe-to-vessel angle remained constant throughout the data acquisition.

\subsection{Other monitoring tests}

Electrocardiographic monitoring and respiratory activity were recorded during experimental runs for control of any effects. Self-perceived anxiety levels by stan- 
dardized questionnaire were recorded before and after data acquisition. Data acquisition were obtained under normal resting oscillations of these parameters. Operator monitoring of subjects' eye, voluntary, and involuntary movements provided a measure of control of these potential sources of artifacts. Motion-related artifacts causing deleterious interferences were marked and deleted from the analysis.

\subsection{Calculations}

Hemispheric advantage was assessed using laterality index $\left(\mathrm{LI}^{\prime}\right)$ expressed as $\mathrm{LI}^{\prime}=($ Right $\mathrm{MBFV}-\mathrm{Left}$ $\mathrm{MBFV} /$ Right MBFV + Left MBFV) $\times 100$. The hemisphere advantage was given by

$\mathrm{LI}=\mathrm{LI}^{\prime}$ paradigm $-\mathrm{LI}^{\prime}$ baseline.

Positive LI suggests either a right lateralization or hemisphere advantage, while negative LI shows left lateralization. Zero LI may suggest no change from baseline or equal bilateral activation.

\subsection{Methodological considerations}

TCD measures MBFV in major intracranial vessels, which has been shown to correlate with blood flow estimates (Taylor, Short, Walker, \& Traystman, 1990). TCD would characterize hemispheric differences in MBFV, but not changes within specific regions of the MCA territory. For example, if areas in the frontal, parietal, and temporal lobes were all crucial for intelligence (as measured by the RPM), activating any one area in the left or right hemisphere would change MBFV in the MCA main stem, and hence lateralization. The reason of course is that, the MCA supplies all these areas. However, the present study focuses on hemispheric advantage, therefore, the application of TCD is most appropriate. The measured MBFV in the main stem of the MCA could be considered as an averaged positive correlate of flow changes related to stimulus effect, suggesting a close correlation with neuronal activity. Moreover, cerebral blood flow velocity changes were elicited by neuronal activity observed during simultaneous TCD and video EEG recordings (Njemanze, Mirza, Rosenfeld, \& Njemanze, 1992). Others have suggested a similar relationship among cerebral blood flow $(\mathrm{CBF})$, metabolism and neuronal activity (Lueck et al., 1989; Petersen, Fox, Snyder, \& Raichle, 1990; Reivich $\&$ Alavi, 1985). The MCA territory supplies about $80 \%$ of blood received by the cerebral hemispheres (Toole, 1984). In relation to site-specific functional localization, TCD could be considered a good test in series with other imaging modalities such as functional magnetic resonance imaging (fMRI) (Schmidt et al., 1999).

Potential sources of motion artifacts were handled by exclusion and cancelling out effects. In pre-experimental control test runs, subtle indication with the finger did not cause a detectable rise in blood flow velocity in both MCAs. However, in the present study, recordings during inter-stimulus interval were marked and removed from the analysis. For male as well as female subjects, the use of right fingers for response was similar to the use of left ( $p>.05$ ). This may suggest that any side-to-side effects of even 'internally planned' or mentally generated movements on data acquisition may have cancelled out. This assumes that the side of mentally generated movement is the same for ANSWER. A sample RPM task was administered in a practice session to eliminate ambiguity in instructions and reduced any possible role of anxiety due to novelty of task. No subject was aware that RPM measured general intelligence. However, all were motivated to solve the tasks to the best of their abilities.

Prior studies have shown that LI computations were independent of partial pressure of end-tidal carbon-dioxide, while the latter affects the sum of right and left MBFV (R + L) (Njemanze, 1991, 1996; Rumsey et al., 1987). The overall accuracy of $R+L$ determines the validity of the measurements of hemispheric advantage in response to a task. It has been argued in the past that a right hemisphere competence may be revealed in nonresponse conditions (Milberg, Whitman, Rourke, \& Glaros, 1981; Segalowitz \& Cohen, 1989) due to absence of motor activity. The stimuli were designed to lack verbalizable features and excluded the possibility of interference from motor activity, by constrained control of subjects' response, until after each measurement.

\subsection{Statistical analysis}

Results were given as mean \pm SD where applicable. The LI scores were analyzed using multivariate analysis of variance (MANOVA). When applicable, group means were compared using $t$ test. All statistical calculations were done using a statistical software package (Statistica, StatSoft, OK, USA).

\section{Results}

The data obtained with means \pm SD and Scheffé test probabilities for post hoc analysis for the different groups are shown in Table 1. The mean Correct score was $50.1 \pm 14 \%$ for all subjects, and the mean for female subjects did not differ from male $(p>.05)$. The design was a two-way MANOVA comprising: two levels of ANSWER (Wrong and Correct) and two levels of GENDER (Female and Male). There was a main effect of GENDER $[F(1,1148)=26.4, p<.0000003$, $M S e=87.6]$. There was no main effect of ANSWER $(p>.05)$. There was a GENDER $\times$ ANSWER interaction $[F(1,1148)=3.8, p<.05 . M S e=12.6]$. As shown in Fig. 1, for Correct ANSWER, females were left 
Table 1

The mean \pm SD and Scheffé test probabilities for post hoc analysis for the different groups

\begin{tabular}{|c|c|c|c|c|c|c|c|}
\hline \multirow[t]{2}{*}{ Answer } & \multirow[t]{2}{*}{ Gender } & \multirow{2}{*}{$\begin{array}{l}\text { Laterality index }(\mathrm{LI}) \\
\text { means } \pm \mathrm{SD}\end{array}$} & \multicolumn{4}{|c|}{ Scheffé test probabilities for post hoc analysis interaction: $1 \times 2$} & \multirow[t]{2}{*}{ Valid $N$} \\
\hline & & & $(1)$ & (2) & (3) & (4) & \\
\hline Wrong & Female & $-0.22 \pm 1.7(1)$ & & 0.16 & 0.36 & 0.007 & 366 \\
\hline Wrong & Male & $0.13 \pm 1.9(2)$ & 0.16 & & 0.002 & 0.7 & 216 \\
\hline Correct & Female & $-0.46 \pm 1.6$ & 0.36 & 0.002 & & 0.000017 & 354 \\
\hline Correct & Male & $0.32 \pm 2.1(4)$ & 0.007 & 0.7 & 0.000017 & & 216 \\
\hline All groups & & $-0.12 \pm 1.8$ & & & & & 1152 \\
\hline
\end{tabular}

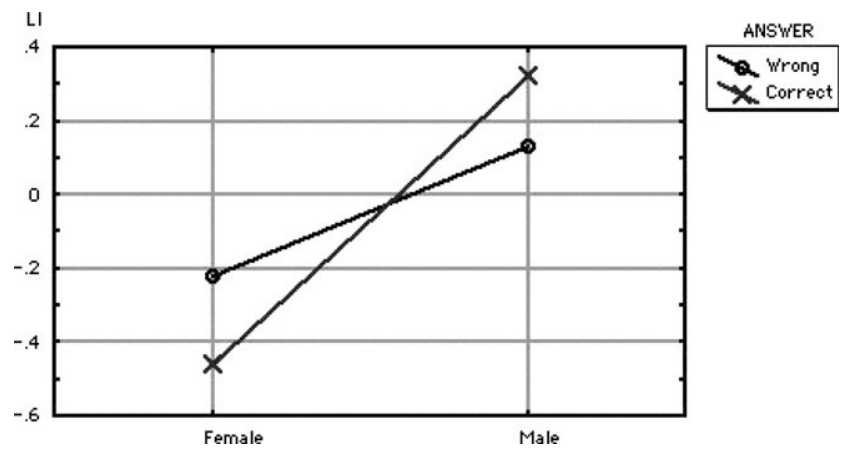

Fig. 1. Two way interaction of GENDER and ANSWER.

lateralized, while males were right lateralized. However, for Wrong ANSWER, there was a tendency towards bilateral hemispheric activation in both female and male subjects.

\section{Discussion}

Overall, these results demonstrate that for successful resolution of RPM tasks, females used a left hemisphere strategy while males used the right hemisphere. This implies that general intelligence is associated with neural systems within one hemisphere that are accessible to a variety of cognitive processes. These findings support the hierarchical hypothesis, and agree with results of recent studies, using positron emission tomography (Duncan et al., 2000) and fMRI (Prabhakaran et al., 1997). Similarly, the results are consistent with earlier studies in patients with lesions in the postrolandic structures (Basso et al., 1973).

In studies using RPM tasks, the areas of regional $\mathrm{CBF}$ activation found in young subjects (Esposito, Kirby, Van Horn, Ellmore, \& Berman, 1999) comprised inferolateral temporal cortex including the fusiform gyrus bilaterally, and the middle temporal gyrus on the left, portions of the left medial temporal cortex including the parahippocampal gyrus, the left inferior parietal lobule, and the cerebellum. These anatomic structures (except for the cerebellum) derive major blood supply from the middle cerebral artery.
Gender differences in cognitive tasks have been a subject of intense debate. The Vandenberg mental rotation tests depicts rotation in three-dimensional space, and typically yields one of the largest established cognitive gender differences favoring males. Furthermore, processing in three dimensions is not a necessary condition for large sex difference on tests of mental rotation (Collins \& Kimura, 1997). Conversely, superior performance by women on task requiring object location memory has challenged the traditional view that men excel on all spatial tasks (Kimura, 1996). The implications of the present findings of gender differential in RPM task could be better understood if a tentative hypothesis is advanced. It could be assumed that general intelligence is related to other lateralized hemispheric cognitive functions including some visuospatial skills. The finding that male subjects used a right hemisphere strategy may well explain the observation in numerous reports, that the average level of spatial talents usually requiring general intelligence is higher in male subjects than female (Buffery \& Gray, 1972). This may suggest that, in males, ipsilateral right hemisphere localization of general intelligence facilitates visuospatial problem-solving. On the other hand, in females, transcallosal pathways are implicated in left hemisphere strategy of visuospatial processing. It is plausible that, reported gender-related hemispheric advantage is dependent on whether task processing strategies implicate either the intra-hemispheric or transcallosal pathways. Studies in commissurotomy patients might provide further insights on the implication of this model.

The finding of bi-hemispheric activation with Wrong ANSWER may suggest that, increasing level of task difficulty is associated with diverse pattern of neural activation due to broad sampling of all major cognitive functions. It could be postulated that, successful RPM problem-solving employs a discrete knowledge strategy (DKS), that selects neural pathways represented in one hemisphere. While unsuccessful outcome implicates a non-discrete knowledge strategy (nDKS).

RPM paradigm could be viewed as a working memory task. Others have argued that the necessity of keeping several conceptual formulations in mind during the 
RPM paradigm is by itself a working memory function (Carpenter et al., 1990). This suggests that the DKS model may have a correlate in mnemonic operations. In other words, DKS model may have a discrete knowledge base (DKB) of essential components needed for task resolution, while for $\mathrm{nDKS}$, DKB is absent, and hence a 'global' or bi-hemispheric search occurs. Further studies with RPM in patients with memory deficits may provide useful insights.

The present study was based on the assumption that both neural and vascular brain structures share similar functional asymmetry. It has been suggested that neocortical vascularization which develops in conjunction with the nervous system and phylogenetic trends may share similar dominance characteristics (Njemanze, 1991). Recent evidence may support this view, for example, a receptor called neuropilin, is present both on neurons, where it is involved in axonal guidance, and on endothelial cells, where it aids angiogenesis (Chen, Chedotal, He, Goodman, \& Tessier-Lavigne, 1997; He \& Tessier-Levigne, 1997; Roush, 1998). Semaphorin III is another protein that has been identified and helps steer axons to their proper destination in the developing nervous system (He \& Tessier-Levigne, 1997). It has been suggested that if neuropilin allows VEGF to act on neurons and semaphorins to act on endothelial cells, this might indicate an unexpected level of coordination between the developing nervous and circulatory systems (Roush, 1998).

The observed gender differences may suggest that sex hormones may be implicated in the above mechanisms. Moreover, there is growing evidence that early exposure to sex hormones has lasting effects on problem-solving behavior, and fluctuations in sex hormones in both men and women are associated with changes in cognitive pattern (Kimura, 1996). Furthermore, animal experiments have demonstrated that in the male rat at birth and in later life, the cortex of several areas in the posterior right hemisphere is significantly thicker than in corresponding areas on the left (Diamond, 1984). By contrast, in the female rat, there are several thicker areas in the left at the same periods. If the male rat is castrated at birth, many of the observed differences are not present at 90 days of age (Diamond, Dowling, \& Johnson, 1981). Similar observations were made in females. In addition, ovarian hormones administered at birth alter the later pattern of cortical asymmetries (Geschwind \& Galaburda, 1985).

Most of the postulates derived from the results of the present study are not aimed at resolving the outstanding issues in intelligence research, but is an attempt to further expand the scope of the discussion raised by results of prior studies (Basso et al., 1973; Duncan et al., 2000). It may appear simplistic to measure intelligence based on a single test. Raven's test is a good measure of intelligence and should account for a good deal of the reasoning in other tests (Carpenter et al., 1990). It may still be that there is simplicity in the present approach. However, it is worthy to note, that even by this singular test, both cerebral lateralization for intelligence and gender differences were observed. It may be useful in future studies using TCD to evaluate if these results can be confirmed when a battery of intelligence tests is used. The present work suggests that TCD would be useful in the study of the diverse issues involved in intelligence research. TCD has high sensitivity (i.e., power to reveal subtle mental performance changes); and diagnosticity (i.e., capability to reveal the underlying processes that lead to these performance changes). The temporal superiority, portability, technical simplicity, cost-effectiveness, reliability, noninvasiveness, and ability to provide a global measure of cerebral lateralization are all added armamentarium that TCD provides to the study of brain cognitive functions.

\section{References}

Basso, A., De Renzi, E., Faglioni, P., Scotti, G., \& Spinnler, H. (1973). Neuropsychological evidence for the existence of cerebral areas critical to the performance of intelligence tasks. Brain, 1996, 96, $715-728$.

Buffery, A. W. H., \& Gray, J. A. (1972). Sex differences in the development of spatial and linguistic skills. In C. Ounsted \& D. C. Taylor (Eds.), Gender differences: Their ontogeny and significance. New York: Churchill Livingston Inc.

Bulla-Hellweg, M., Vollmer, J., Götzen, A., Skreczek, W., \& Hartje, W. (1996). Hemispheric asymmetry of arterial blood flow velocity changes during verbal and visuospatial tasks. Neuropsychologia, 34, 987-991.

Carpenter, P. A., Just, M. A., \& Shell, P. (1990). What one intelligence test measures: A theoretical account of the processing in the Raven progressive matrices test. Psychological Review, 97, $404-431$.

Chen, H., Chedotal, A., He, Z., Goodman, C. S., \& Tessier-Lavigne, M. (1997). Neuropilin-2, a novel member of the neuropilin family, is a high affinity receptor for the samaphorins Sema E and Sema IV but not Sema II. Neuron, 19, 547-559.

Collins, D. W., \& Kimura, D. (1997). A larger sex difference on a two dimensional mental rotation task. Behavioral Neuroscience, 111, 845-849.

Cupini, L. M., Matteis, M., Troisi, E., Sabbadini, M., Bernardi, G., Caltagirone, C., \& Silvestrini, M. (1996). Bilateral simultaneous transcranial Doppler monitoring of flow velocity changes during visuospatial and verbal working memory tasks. Brain, 119, $1249-1253$.

Diamond, M. C. (1984). Age, sex, and environmental influences. In N. Geschwind \& A. M. Galaburda (Eds.), Cerebral dominance: The biological foundations (pp. 134-146). Cambridge, MA: Harvard University Press.

Diamond, M. C., Dowling, G. A., \& Johnson, R. E. (1981). Morphological cerebral cortical asymmetry in male and female rats. Experimental Neurology, 71, 261-268.

Droste, D. W., Harders, A. G., \& Rastogi, E. (1989). Two transcranial Doppler studies on blood flow velocity in both middle cerebral arteries during rest and the performance of cognitive tasks. Neuropsychologia, 27, 1221-1230.

Duncan, J., Seitz, R. J., Kolodny, J., Bor, D., Herzog, H., Ahmed, A., Newell, F. N., \& Emslie, H. (2000). A neural basis for general intelligence. Science, 289, 457-460. 
Esposito, G., Kirby, B. S., Van Horn, J. D., Ellmore, T. M., \& Berman, K. F. (1999). Context-dependent, neural system-specific neurophysiological concomitants of ageing: mapping PET correlates during cognitive activation. Brain, 122, 963-979.

Evers, S., Dannert, J., Rodding, D., Rotter, G., \& Ringelstein, E. B. (1999). The cerebral haemodynamics of music perception. A transcranial Doppler sonography study. Brain, 122, 75-85.

Geschwind, N., \& Galaburda, A. M. (1985). Cerebral lateralization. Biological mechanisms, associations, and pathology: II.A hypothesis and a program for research. Archives of Neurology, 42, $521-552$.

Hartje, W., Ringelstein, E. B., Kistinger, B., Fabianek, D., \& Willmes, K. (1994). Transcranial Doppler ultrasonic assessment of middle cerebral artery blood flow velocity changes during verbal and visuospatial cognitive tasks. Neuropsychologia, 32, 1443-1452.

He, Z., \& Tessier-Levigne, M. (1997). Neuropilin is a receptor for axonal chemorepellent Semaphorin. Cell, 90, 739-751.

Jensen, A. R. (1987). The g beyond factor analysis. In A. R. Ronning, J. A. Glover, J. C. Conoley, \& J. C. Witt (Eds.), The influence of cognitive psychology on testing (pp. 87-142). Hillsdale, NJ: Erlbaum.

Kelley, R. E., Chang, J. Y., Scheinman, N. J., Levin, B. E., Duncan, R. C., \& Lee, S.-C. (1992). Transcranial Doppler assessment of cerebral flow velocity during cognitive tasks. Stroke, 23, 9-14.

Kimura, D. (1996). Sex, sexual orientation and sex hormones influence human cognitive function. Current Opinion in Neurobiology, 6, 259-263.

Knecht, S., Deppe, M., Ringelstein, E. B., Wirtz, M., Lohmann, H., Drager, B., Huber, T., \& Henningsen, H. (1998). Reproducibility of functional transcranial Doppler sonography for determining hemispheric language lateralization. Stroke, 29, 1155-1159.

Lashley, K. S. (1921). Studies of cerebral function in learning. The effect of long continued practice upon cerebral localization. Journal of Comparative Psychology, 1, 452-468.

Lashley, K. S. (1929). Brain Mechanisms and Intelligence. Hillsdale, NJ: University of Chicago Press.

Lueck, C. J., Zeki, S., Friston, K. J., Deiber, M. P., Cope, P., Cunningham, V. J., Lammertsma, A. A., Kennerd, C., \& Frackowiak, R. S. J. (1989). The colour center in the cerebral cortex of man. Nature, 340, 386-389.

Markus, H. S., \& Boland, M. (1992). Cognitive activity monitored by non-invasive measurement of cerebral blood flow velocity and its application to the investigation of cerebral dominance. Cortex, 28, $575-581$.

Milberg, W. P., Whitman, R. D., Rourke, D., \& Glaros, A. G. (1981). Role of sub-vocal motor activity in dichotic speech perception and selective attention. Journal of Experimental Psychology: Human Perception Performance, 7, 231-239.

Njemanze, P. C. (1991). Cerebral lateralization in linguistic and nonlinguistic perception: Analysis of cognitive styles in the auditory modality. Brain and Language, 41, 367-380.

Njemanze, P. C. (1996). Cerebral lateralization in random letter task in the visual modality: A transcranial Doppler study. Brain and Language, 63, 315-325.

Njemanze, P. C. (2002). Cerebral lateralization for motor tasks in simulated microgravity. A transcranial Doppler technique for astronauts. Journal of Gravitational Physiology, 9, 33-34.

Njemanze, P.C. (2004). Asymmetry in cerebral blood flow velocity with processing of facial images during head-down rest. Aviation Space and Environmental Medicine (in press).

Njemanze, P. C., Gomez, C. R., \& Horenstein, S. (1992). Cerebral lateralization and color perception: A transcranial Doppler study. Cortex, 28, 69-75.

Njemanze, P. C., Mirza, W. U., Rosenfeld, W. E., \& Njemanze, V. U. (1992). Cerebral blood flow velocity and EEG amplitude in generalized seizures: A case study. Journal of Neuroimaging, 2, $151-155$.
Oldfield, R. C. (1971). The assessment and analysis of handedness: The Edinburgh Inventory. Neuropsychologia, 9, 97-114.

Petersen, S. E., Fox, P. T., Snyder, A. Z., \& Raichle, M. E. (1990). Activation of extrastriate and frontal cortical areas by visual words and word-like stimuli. Science, 249, 1041-1044.

Prabhakaran, V., Smith, J. A., Desmond, J. E., Glover, G. H., \& Gabrieli, J. D. (1997). Neural substrates of fluid reasoning: an fMRI study of neocortical activation during performance of the Raven's Progressive Matrices Test. Cognitive Psychology, 33, $43-63$.

Raven, J. C. (1938). Guide to progressive matrices. London: H.K. Lewis.

Reivich, M., \& Alavi, A. (1985). Effects of psychophysiological stimuli on local cerebral glucose consumption in humans. In The metabolism of human brain studies with positron emission tomography (pp. 305-313). New York: Raven Press.

Rihs, F., Gutbrod, K., Steiger, H. J., Sturzenegger, M., \& Mattle, H. P. (1995). Determination of cognitive hemispheric dominance by "stereo" transcranial Doppler sonography. Stroke, 26, 7073.

Roush, W. (1998). Receptor link blood vessels, axons. Science, 279, 2042.

Rumsey, J. M., Berman, K. F., Denckla, M. B., Hamburger, S. D., Kruesi, M. J., \& Weinberger, D. R. (1987). Regional cerebral blood flow in severe developmental dyslexia. Archives of Neurology, 44, 114-1155.

Satz, P., Strauss, E., \& Whitaker, H. (1990). The ontogeny of hemispheric specialization: Some old hypothesis revisited. Brain and Language, 38, 596-614.

Schmidt, P., Krings, T., Willmes, K., Roessler, F., Reul, J., \& Thron, A. (1999). Determination of cognitive hemispheric lateralization by "functional" transcranial Doppler cross-validated by functional MRI. Stroke, 30, 939-945.

Segalowitz, S. J., \& Cohen, H. (1989). Right hemisphere EEG sensitivity to speech. Brain and Language, 37, 220-231.

Silvestrini, M., Troisi, E., Matteis, M., Razzano, C., \& Caltagirone, C. (1994). Correlations of flow velocity changes during mental activity and recovery from aphasia in ischemic stroke. Neurology, 50, 191-195.

Snow, R. E., Kyllonen, P. C., \& Marshalek, B. (1984). The topography of ability and learning correlations. In R. J. Sternberg (Ed.), Advances in the psychology of human intelligence. Hillsdale, NJ: Erlbaum.

Spearman, C. E. (1904). General intelligence, objectively determined and measured. American Journal of Psychology, 15, 201-293.

Spearman, C. E. (1923). The nature of intelligence and the principles of cognition. London: Macmillian.

Spearman, C. E. (1927). The abilities of man. New York: Macmillian.

Taylor, G. A., Short, B. L., Walker, L. K., \& Traystman, R. L. (1990). Intracranial blood flow: Quantification with duplex Doppler and color Doppler flow. Radiology, 176, 231-236.

Thomson, G. H. (1951). The factorial structure of human ability. London: University of London Press.

Thurstone, L. L. (1938). Primary mental abilities. Chicago, IL: University of Chicago Press.

Thurstone, L. L. (1940). The vectors of mind. Chicago: University of Chicago Press.

Toole, J. F. (1984). Cerebrovascular disease. New York: Raven Press p. 9.

Varnadore, A. E., Roberts, A. E., \& McKinney, W. M. (1997). Modulations in cerebral hemodynamics under three response requirements while solving language-based problems: A transcranial Doppler study. Neuropsychologia, 35, 1209-1214.

Vingerhoets, G., \& Stroobant, N. (1999). Lateralization of cerebral blood flow velocity changes during cognitive tasks. A simultaneous bilateral transcranial Doppler study. Stroke, 30, 2152-2158. 